\title{
Nouvelles du Comité central
}

\author{
Jeanine Glarner \\ Spécialiste en communication, division Communication de la FMH
}

Peer reviews en psychiatrie - A l'initiative de l'Alliance Peer Review CH (FMH, H+, Swiss Nurse Leaders), un projet-pilote a été mené en 2014/15 dans le but de promouvoir des peer reviews uniformes et interprofessionnels dans les soins aigus au plan national. Forte des expériences de ce projet-pilote, l'Alliance juge pertinent de standardiser la procédure à l'échelle nationale également dans le domaine de la psychiatrie. Actuellement, aucune initiative nationale ou démarche comparable n'existe dans ce domaine. Des propositions dans ce sens doivent donc être élaborées en collaboration avec des experts issus de la médecine, des soins infirmiers et du secteur thérapeutique. Le Comité central soutient ce projet.

Profil d'intégration axé sur les ressources (PIR) - Lors d'une incapacité de travail d'un collaborateur, la coordination et la collaboration entre employeur, médecin traitant et assurances sociales jouent un rôle essentiel dans le succès durable de la réinsertion. Pour cela, l'échange entre médecin, patient et employeur est primordial. L'association Compasso, qui regroupe employeurs, médecins et Confédération, a développé un profil d'intégration axé sur les ressources (PIR) pour favoriser cet échange et permettre une meilleure évaluation de la capacité de travail. A l'aide d'un certificat médical détaillé tenant compte des exigences relatives au poste occupé, le médecin traitant peut évaluer précisément si et dans quelle mesure son patient peut encore exercer, et quelles conditions et mesures de soutien sont nécessaires pour l'intégrer sur son lieu de travail. Idéalement, le médecin devrait connaître les exigences et les conditions cadres de l'employeur, c'est pourquoi un échange soutenu entre médecin, patient et employeur doit être encouragé. Le Comité central soutient cette initiative.

Jeanine Glarner

Elfenstrasse 18

CH-3000 Berne 15

Tél. 0313591111

Fax 0313591112

kommunikation[at]fmh.ch tons ont fixé, dans la stratégie nationale en matière de démence, des objectifs destinés à améliorer la qualité de vie de ce groupe de patients en constante augmentation, de réduire la charge liée à la maladie et d'assurer la qualité de la prise en charge. De son côté, l'Académie suisse des sciences médicales (ASSM) a rédigé des directives médico-éthiques "Prise en charge et traitement des personnes atteintes de démence». Le Comité central propose à la Chambre médicale de mai 2018 de reprendre ces directives dans le Code de déontologie.

Stratégie Cybersanté Suisse 2.0 - Avec l'adoption de la Stratégie Suisse numérique, le Conseil fédéral a mandaté l'élaboration d'une stratégie Cybersanté Suisse visant à mettre à jour la stratégie existante et à l'élargir en prévision de l'introduction du dossier électronique du patient (DEP). Le projet de stratégie en question entretient cependant la confusion entre stratégie DEP et stratégie de numérisation dans le système de santé suisse. La promotion du DEP ne peut avoir lieu que si tous les médecins sont connectés. Or, le champ d'action «Encourager la numérisation» précise uniquement que la Confédération et les cantons encouragent la numérisation dans le cadre de leurs responsabilités respectives en remplaçant systématiquement les processus papier par des processus numériques. Aucune condition ni mesure apte à favoriser le passage au numérique des médecins dans le secteur ambulatoire n'est précisée. En outre, des éléments centraux font défaut, comme l'accès aux nouvelles technologies et leur utilisation dans le système de santé, l'impact de la numérisation sur la société ou sur les processus et l'organisation du travail dans le secteur de la santé. Une stratégie en matière de cybersanté doit mettre en évidence les risques liés à un tel changement structurel. Dans sa prise de position, le Comité central demande donc un remaniement en profondeur de la stratégie Cybersanté et la distinction entre la stratégie visant à encourager le passage au numérique dans la santé et celle visant à promouvoir et à diffuser le DEP. 